\title{
Superdirective Magnetic Dipole Array as a First-Order Probe for Spherical Near-Field Antenna Measurements
}

Kim, Oleksiy S.; Pivnenko, Sergey; Breinbjerg, Olav

Published in:

I E E E Transactions on Antennas and Propagation

Link to article, DOI:

10.1109/TAP.2012.2207363

Publication date:

2012

Document Version

Early version, also known as pre-print

Link back to DTU Orbit

Citation (APA):

Kim, O. S., Pivnenko, S., \& Breinbjerg, O. (2012). Superdirective Magnetic Dipole Array as a First-Order Probe for Spherical Near-Field Antenna Measurements. I E E E Transactions on Antennas and Propagation, 60(10). https://doi.org/10.1109/TAP.2012.2207363

\section{General rights}

Copyright and moral rights for the publications made accessible in the public portal are retained by the authors and/or other copyright owners and it is a condition of accessing publications that users recognise and abide by the legal requirements associated with these rights.

- Users may download and print one copy of any publication from the public portal for the purpose of private study or research

- You may not further distribute the material or use it for any profit-making activity or commercial gain

- You may freely distribute the URL identifying the publication in the public portal 


\title{
Superdirective Magnetic Dipole Array as a First-Order Probe for Spherical Near-Field Antenna Measurements
}

\author{
Oleksiy S. Kim, Sergey Pivnenko, Member, IEEE, and Olav Breinbjerg, Member, IEEE
}

\begin{abstract}
The theory as well as numerical and experimental results are presented for a superdirective array composed of closely spaced electrically small resonant magnetic dipole elements. The array operates on a metal ground plane and can exhibit a maximum directivity of $11.5 \mathrm{dBi}, 15.2 \mathrm{dBi}$, and $17.8 \mathrm{dBi}$ (including $3 \mathrm{~dB}$ due to the ground plane), for 2,3 , and 4 magnetic dipoles, respectively. The array is self-resonant and is directly excited by a 50-ohm coaxial cable through the ground plane. The array radiates essentially the $|\mu|=1$ spherical modes, which, despite a narrow bandwidth, makes it an excellent firstorder probe for spherical near-field antenna measurements at low frequencies.
\end{abstract}

Index Terms-Electrically small antennas, supergain array, superdirectivity, magnetic dipole, spherical near-field antenna measurements, first-order probe

\section{INTRODUCTION}

$\mathbf{I}$ $\mathrm{N}$ spherical near-field antenna measurements, the measured signal is influenced by the probe, which necessitates a probe correction in the near-field to far-field transformation [1]. This can be done most efficiently with a first-order probe, that is a probe radiating fields with $|\mu|=1$ azimuthal variation only. Although probe correction techniques for higher-order probes have recently been developed [2]-[4], they are more demanding in terms of the computational complexity as well as in terms of calibration and post-processing time.

The commonly used first-order probes for spherical nearfield antenna measurements are conical horns fed by a circular waveguide operating in the fundamental $\mathrm{TE}_{11}$-mode regime. The waveguides are made long enough so that they function as effective filters for higher-order modes inevitably excited at their feed section. Thus, a radiation pattern with a high purity of the $|\mu|=1$ azimuthal mode is achieved. Unfortunately, these probes become bulky and heavy as frequency decreases, and already at $1 \mathrm{GHz}$ a circular waveguide probe is challengingly large. For example, the largest first-order probe at the DTU-ESA Spherical Near-Field Antenna Test Facility [5] operates in the frequency band $1.4-1.65 \mathrm{GHz}$ and weighs $12 \mathrm{~kg}$.

The use of light-weight first-order probes based on the electrically small antenna concept to cover discrete frequencies

This work is supported by the European Space Agency (ESA) within the project 'Higher-Order Near-Field Probes (HONFP)', ESTEC contract No.22812/09/NL/JD/al.

The authors are with the Department of Electrical Engineering, Electromagnetic Systems, Technical University of Denmark, DK-2800 Kgs. Lyngby, Denmark (e-mail: osk@elektro.dtu.dk) below $1 \mathrm{GHz}$ was first envisaged in [6]. The underlying

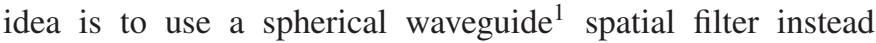
of the cylindrical one. If in a circular waveguide the cut-off frequency for a particular mode is dictated by the waveguide radius, in a spherical waveguide the radius of the minimum sphere enclosing the source currents governs the mode content of the radiated fields. It can be shown, using the spherical wave theory, that the sources confined to an electrically small volume radiate essentially the elementary dipole fields, that is the fields with $\nu=1$ polar variation and $|\mu| \leq 1$ azimuthal variation [1].

On the other hand, the directivity of a usual electrically small antenna is only $1.76 \mathrm{dBi}$, whereas the directivity of a probe should be much higher to effectively suppress specular reflections from the walls of the anechoic chamber [7] and to reduce the influence of the measurement tower supporting the probe. The problem can be overcome by arranging electrically small antenna elements in a superdirective array. As first shown by Uzkov [8], the maximum end-fire directivity of a linear array of $N_{\Sigma}$ isotropic (acoustic) radiators approaches $N_{\Sigma}^{2}$, as the distance between them vanishes. For an array of elementary dipoles (electric or magnetic), the expression for the directivity is not so explicit; the maximum end-fire directivities of arrays of 2, 3, and 4 dipoles in free space are numerically found to be $7.2 \mathrm{dBi}, 10.3 \mathrm{dBi}$, and $12.7 \mathrm{dBi}$, respectively [9]. Furthermore, if the electrically small elements in the array are resonant, they do not all need to be actively fed. It is sufficient to have a single active element, while short-circuiting the others to let them operate as passive directors/reflectors in a Yagi-Uda-like manner [10], [11].

To our knowledge, all numerical and experimental results on superdirective linear arrays of electrically small resonant elements presented in the literature so far are related to electric monopoles on a metal ground plane [10]-[13]. In such arrays, the maximum directivity occurs along the ground plane, and thus, these configurations cannot be applied as probes in spherical near-field antenna measurements. Obviously, an endfire array can also be composed of electric dipoles, but this configuration would require a balanced feed as well as an effective suppression of the back radiation to isolate the array from the mounting structure at the measurement tower. This can be done using either effective absorbers or a ground plane. In the latter case, the array should be placed normal to and

\footnotetext{
${ }^{1}$ The term 'spherical waveguide' is usually applied to a source-free homogeneous region, where the electromagnetic problem is treated using the spherical wave theory.
} 
a quarter of wavelength away from the ground plane, which would make the design quite cumbersome.

Therefore, we follow an alternative approach and consider magnetic dipoles arranged in a superdirective array. In this case, an actively fed magnetic dipole can be placed directly on a metal ground plane with another magnetic dipole acting as a director right in front of the first one. As we show later in this paper (Section II), such an array of elementary magnetic dipoles can exhibit a maximum directivity of $11.5 \mathrm{dBi}$ (including $3 \mathrm{~dB}$ due to the ground plane), which is enough for a probe to perform optimally in the anechoic chamber of the DTUESA Spherical Near-Field Antenna Test Facility [7]. This configuration has also the practical advantage that it allows the active element to be fed directly through the ground plane, thus avoiding extra structures interfering with the antenna.

Thus, the purpose of this paper is two-fold. First, it demonstrates, both theoretically and experimentally, how a superdirective array of electrically small resonant magnetic dipole elements can be designed. Second, it shows that this array can exhibit a high degree of $|\mu|=1$ azimuthal mode purity and thus can be employed as a first-order probe for spherical near-field antenna measurements.

The paper is organized as follows. In Section II, we find the maximum directivity for a closely spaced array of identical radiators over a ground plane. In Section III, we choose an appropriate electrically small resonant magnetic dipole element and investigate its spherical mode content. In Section IV, an array composed of two of these elements is studied numerically. A prototype and the measured characteristics of a superdirective first-order probe are presented and discussed in Section V. Finally, we summarize the results in Section VI.

\section{MaXimum Directivity of Symmetric Linear ARRAY OF IDENTICAL RADIATORS}

To find the maximum possible directivity of a linear array of $(N+1)$ discrete radiators over a ground plane, we apply the procedure by Uzkov [8] to an equivalent symmetric array constructed from the original one using the image principle.

Consider a linear array of $N_{\Sigma}=(2 N+1)$ identical radiators arranged along the $z$-axis in a Cartesian coordinate system, such that the central radiator is located at the origin. The radiators are equally spaced by a distance $d$ and succesively numbered from $-N$ to $N$. We enforce a symmetry with respect to the $x y$-plane implying that the complex excitation amplitudes of the radiators $A_{n}$ satisfy the relation

$$
A_{n}=A_{-n}, \quad n=1,2, \ldots N .
$$

Consequently, the far-field radiation pattern of the array can be written as

$$
\mathbf{F}(\theta, \phi)=\sum_{n=0}^{N} \delta_{n} A_{n} \mathbf{f}(\theta, \phi) \cos (n k d \cos \theta)
$$

where

$$
\delta_{n}= \begin{cases}1, & \text { for } n=0 \\ 2, & \text { for } n>0\end{cases}
$$

and $k$ is the free-space wave number. We assume that the radiation pattern of each radiator $\mathbf{f}(\theta, \phi)$ does not change with change of the excitation amplitudes.

The directivity of the array is then given as

$$
\begin{aligned}
& D(\theta, \phi)=\frac{4 \pi|\mathbf{F}(\theta, \phi)|^{2}}{\int_{0}^{2 \pi} \int_{0}^{\pi}|\mathbf{F}(\theta, \phi)|^{2} \sin \theta d \theta d \phi} \\
& =\frac{\sum_{m=0}^{N} \sum_{n=0}^{N} a_{m} a_{n}^{*} \cos (m k d \cos \theta) \cos (n k d \cos \theta)}{\sum_{m=0}^{N} \sum_{n=0}^{N} a_{m} a_{n}^{*} h_{m n}(\theta, \phi)}
\end{aligned}
$$

where $a_{n}=\delta_{n} A_{n}|\mathbf{f}(\theta, \phi)|$ and

$$
\begin{aligned}
& h_{m n}(\theta, \phi)=\frac{H_{m n}}{|\mathbf{f}(\theta, \phi)|^{2}} \\
& =\frac{\int_{0}^{2 \pi} \int_{0}^{\pi}|\mathbf{f}(\theta, \phi)|^{2} \cos (m k d \cos \theta) \cos (n k d \cos \theta) \sin \theta d \theta d \phi}{4 \pi|\mathbf{f}(\theta, \phi)|^{2}}
\end{aligned}
$$

We are looking for a set of coefficients $a_{0 n}$ that maximizes $D(\theta, \phi)$ in the array end-fire directions: $\theta_{0}=0$ and, due to the enforced symmetry, $\theta=\pi\left(\phi_{0}=0\right)$. According to [14], the coefficients $a_{0 n}$ maximizing the ratio of the form (3b) can be found by solving the following system of linear equations

$$
\sum_{n=0}^{N} a_{0 n} h_{m n}\left(\theta_{0}, \phi_{0}\right)=\cos \left(m k d \cos \theta_{0}\right), \quad m=1,2, \ldots N .
$$

The maximum directivity is then

$$
D_{\text {max }}=D_{0}\left(\theta_{0}, \phi_{0}\right)=\sum_{n=0}^{N} a_{0 n} \cos \left(n k d \cos \theta_{0}\right) .
$$

In the following, we consider two particular cases of symmetric arrays - with isotropic and dipole radiators, respectively.

\section{A. Isotropic Radiators}

Using the procedure outlined in [9], it can be shown that the radiation pattern of a symmetric array of isotropic (acoustic) radiators with $|\mathbf{f}(\theta, \phi)|=1$ can be written as

$$
F(\theta, \phi)=\sum_{n=0}^{N} c_{n} P_{2 n}(\cos \theta), \quad \text { as } k d \rightarrow 0
$$

where $c_{n}$ are complex coefficients. Due to the enforced symmetry, the sum in (7) contains only even-order Legendre polynomials $P_{2 n}(\cos \theta)$.

Similar to (5) and (6), we find

$$
\begin{gathered}
c_{n}=(4 n+1) P_{2 n}\left(\cos \theta_{0}\right) \\
D_{\max }=\sum_{n=0}^{N}(4 n+1)\left(P_{2 n}\left(\cos \theta_{0}\right)\right)^{2} .
\end{gathered}
$$

Finally, in the end-fire directions $\left(\theta_{0}=0\right.$ and $\pi$ ), the maximum directivity becomes

$$
D_{\max }=\sum_{n=0}^{N}(4 n+1)=(N+1)(2 N+1)=\frac{N_{\Sigma}^{2}}{2}+\frac{N_{\Sigma}}{2}
$$


We note, that the maximum end-fire directivity (10) of the symmetric array approaches half of the Uzkov's limit $\left(N_{\Sigma}^{2}\right)[8]$ for a non-symmetric case (where the directivity is maximized in one end-fire direction), as the number of radiators $N_{\Sigma}$ increases.

\section{B. Dipole Radiators}

For an elementary electric or magnetic dipole, whose moment is aligned along the $y$-axis, the far-field radiation pattern can be written as

$$
|\mathbf{f}(\theta, \phi)|^{2}=\cos ^{2} \phi+\cos ^{2} \theta \sin ^{2} \phi .
$$

Substituting (11) into (4), we obtain

$$
\begin{aligned}
& H_{m n}= \frac{1}{2} \sum_{ \pm}\left\{\frac{\sin [(m \pm n) k d]}{(m \pm n) k d}\left(1-\frac{1}{[(m \pm n) k d]^{2}}\right)\right. \\
&\left.+\frac{\cos [(m \pm n) k d]}{[(m \pm n) k d]^{2}}\right\}, \quad \text { for } m \neq n \\
& H_{m n}= \frac{1}{3}+\frac{\sin (2 n k d)}{4 n k d}\left(1-\frac{1}{(2 n k d)^{2}}\right)+\frac{\cos (2 n k d)}{2(2 n k d)^{2}} \\
& \text { for } m=n \neq 0
\end{aligned}
$$

and $H_{m n}=2 / 3$ for $m=n=0$.

By using (12) in (5), we set up a system of linear equations, whose solution give us the coefficients $a_{n}$ ensuring the maximum directivity (6). We numerically found that symmetric arrays of $N_{\Sigma}=3,5$, and 7 elementary dipoles exhibit the end-fire directivity (at $\theta_{0}=0$ and $\pi$ ) of $8.5 \mathrm{dBi}, 12.2 \mathrm{dBi}$, and $14.8 \mathrm{dBi}$, respectively, as the spacing between the dipoles vanishes $^{2}(k d \rightarrow 0)$.

The described scenario and the obtained results apply to magnetic dipoles over a perfectly electrically conducting (PEC) ground plane and electric dipoles over a perfectly magnetically conducting (PMC) ground plane. In these cases, $3 \mathrm{~dB}$ due to the ground plane should be added to the directivities given above, resulting in $11.5 \mathrm{dBi}, 15.2 \mathrm{dBi}$, and $17.8 \mathrm{dBi}$ for the corresponding arrays of 2, 3, and 4 dipoles over a ground plane, respectively. Thus, an array of two electrically small magnetic dipole elements over a metal ground plane should provide the directivity ample for our measurement probe.

\section{The ELEMENT FIRST}

To realize the envisaged superdirective first-order probe, we first identify and investigate a single magnetic dipole element that is able to radiate a pure $|\mu|=1$ azimuthal mode. It appears that not all electrically small loop-like elements are

\footnotetext{
${ }^{2}$ As in the case of isotropic radiators (Section II-A), the maximum directivity of the symmetric array of elementary dipoles comes close to half of the maximum directivity possible for a unidirectional array of the same topology. Adding $3 \mathrm{~dB}$ (due to a ground plane) to the directivity of the former results in a value just above the directivity of the latter; and the difference between the two decreases with increasing number of radiators For example, compare $10.3 \mathrm{dBi}, 14.5 \mathrm{dBi}$, and $17.3 \mathrm{dBi}$ maximum directivity (obtained using Uzkov's procedure [8], [11]) of the unidirectional arrays of 3 , 5 , and 7 elementary dipoles (electric or magnetic) in free space, respectively, with $11.5 \mathrm{dBi}, 15.2 \mathrm{dBi}$, and $17.8 \mathrm{dBi}$ for the 2-, 3-, and 4-element arrays of elementary magnetic (electric) dipoles over a PEC (PMC) ground plane, respectively.
}

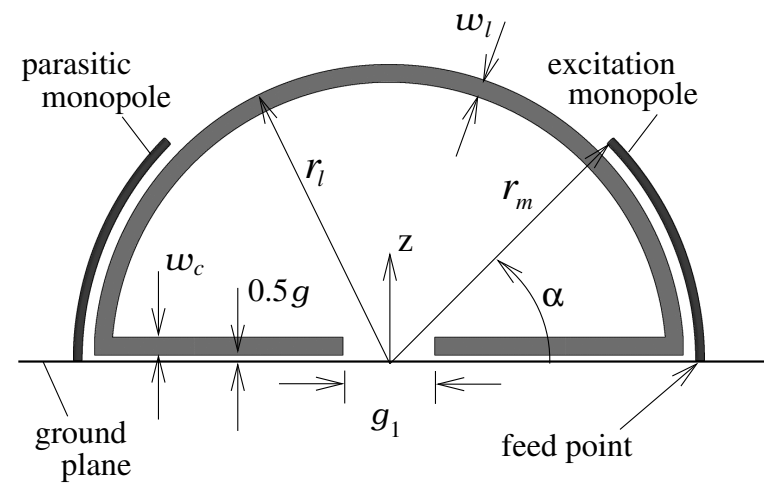

Fig. 1. Electrically small resonant magnetic dipole element: Capacitively loaded loop (CLL) excited by a curved monopole on a metal ground plane. The parasitic monopole is added to suppress even azimuthal spherical modes.

able to fulfill this stringed requirement. Indeed, electrically small dimensions of an element facilitate the suppression of $\nu>1$ - and consequently $|\mu|>1$ - modes. However, any asymmetry of the electric current with respect to two orthogonal vertical planes manifests itself in rising $|\mu| \neq 1$ modes, in particular $|\mu|=0$ modes. Therefore, there must be ensured a symmetry of the electric current with respect to the vertical planes.

Based on these considerations, we have selected a symmetric two-gap version of capacitively loaded loop (CLL) [15] to be a building block for our first-order probe. This particular shape consisting of a loop loaded with two symmetric parallel strip capacitors was used, for example, in [16] to construct a Huygens source.

We put the CLL on a metal ground plane and use a curved monopole to excite it (Fig. 1), similar to the split-ring resonator antenna reported in [17]. This excitation technique provides a great flexibility with respect to adjustment of the antenna input impedance. As shown in [17], [18], the input resistance at resonance can be varied in a wide range by changing the length of the curved monopole.

The reflection symmetry would not be complete without a parasitic curved monopole added to the opposite side of the CLL and short-circuited to the ground plane, as shown in Fig. 1. The effect is illustrated in Fig. 2, where the relative radiated power of spherical modes with azimuthal indices $|\mu|$ from 0 to 3 is plotted for two cases - with and without the parasitic monopole. As expected, the presence of the parasitic monopole significantly reduces the contribution of even azimuthal modes in the antenna spherical wave spectrum. The relative power of the $\mu=0$ modes is reduced by $20 \mathrm{~dB}$, while the $|\mu|=2$ modes become totally negligible. At the same time, we note that the odd $|\mu|=3$ modes are not affected.

In the example above, the radius of CLL is $r_{l}=53 \mathrm{~mm}$, the width of the loop and the capacitor strips is $w_{l}=w_{c}=3 \mathrm{~mm}$, the gap height is $g=2 \mathrm{~mm}$, and the gap width between the strips forming the capacitors is $g_{1}=29.5 \mathrm{~mm}$. The active and parasitic monopoles are modeled as curved wire segments with radius $0.5 \mathrm{~mm}$ and length measured in angular units $\alpha=34^{\circ}$. The monopoles are located at radius $r_{m}=54 \mathrm{~mm}$, while the active monopole is excited by a delta-gap voltage generator. 


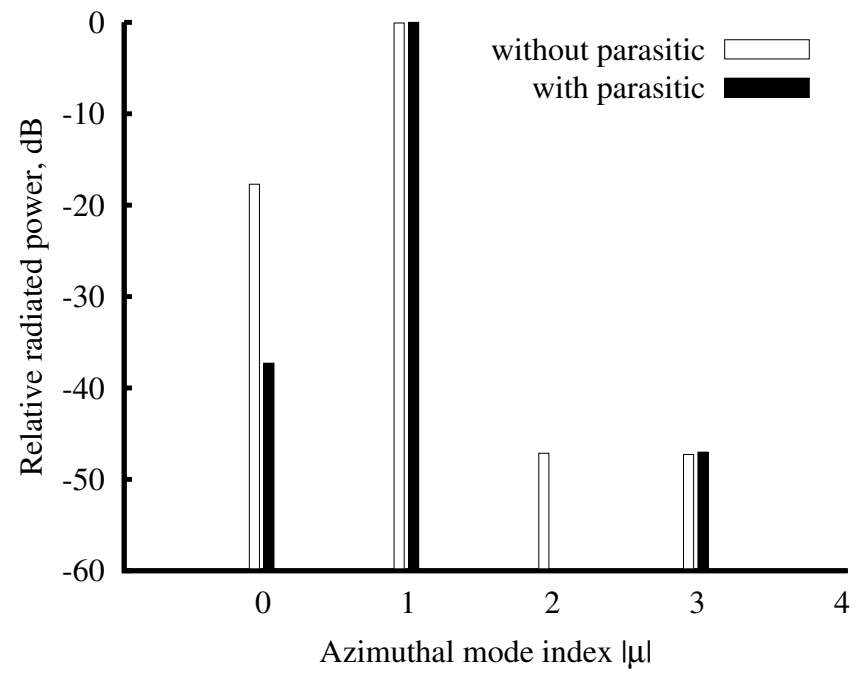

Fig. 2. Spectra of azimuthal spherical modes of the electrically small resonant magnetic dipole element (Fig. 1) with and without parasitic monopole.

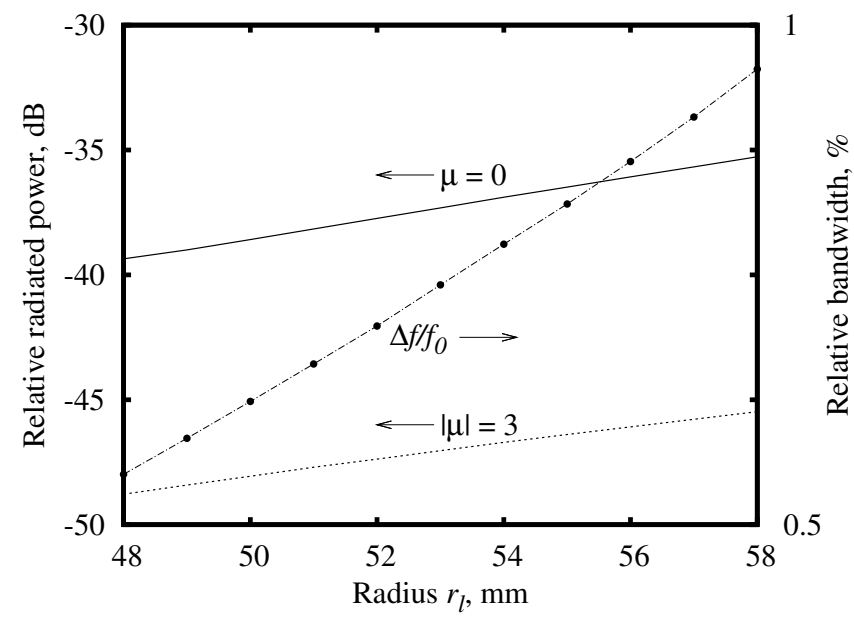

Fig. 3. Relative radiated power of $\mu=0$ and $|\mu|=3$ spherical modes and relative frequency bandwidth versus the radius of the electrically small resonant magnetic dipole element (Fig. 1).

The antenna is self-resonant and matched to $50 \mathrm{ohms}$ at $f_{0}=435 \mathrm{MHz}$. The solution to the problem is obtained using the surface integral equation technique [19] assuming that all conductors are PEC.

Obviously, dimensions of the antenna should be as large as possible to ensure the largest possible frequency bandwidth. On the other hand, increasing the dimensions of the source - in this case, the radius of the CLL $r_{l}$ - leads to rise in higher-order modes. Fig. 3 shows that the relative $-10 \mathrm{~dB}$ bandwidth increases by $75 \%$ as the radius $r_{l}$ increases from $48 \mathrm{~mm}$ to $58 \mathrm{~mm}$ (the radius of the curved monopoles is always $r_{m}=r_{l}+1 \mathrm{~mm}$, while the gap width $g_{1}$ and the length of the curved monopole $\alpha$ are adjusted to retain the resonance at $435 \mathrm{MHz}$ and the input impedance at $50 \mathrm{ohms}$ ). At the same time, the relative power of the $|\mu|=3$ modes increases by more than $3 \mathrm{~dB}$.

Notably, the $\mu=0$ modes are almost $10 \mathrm{~dB}$ higher than the $|\mu|=3$ modes, and they also grow as the radius $r_{l}$ increases (Fig. 3). Whereas the growth of the $|\mu|=3$ modes can be

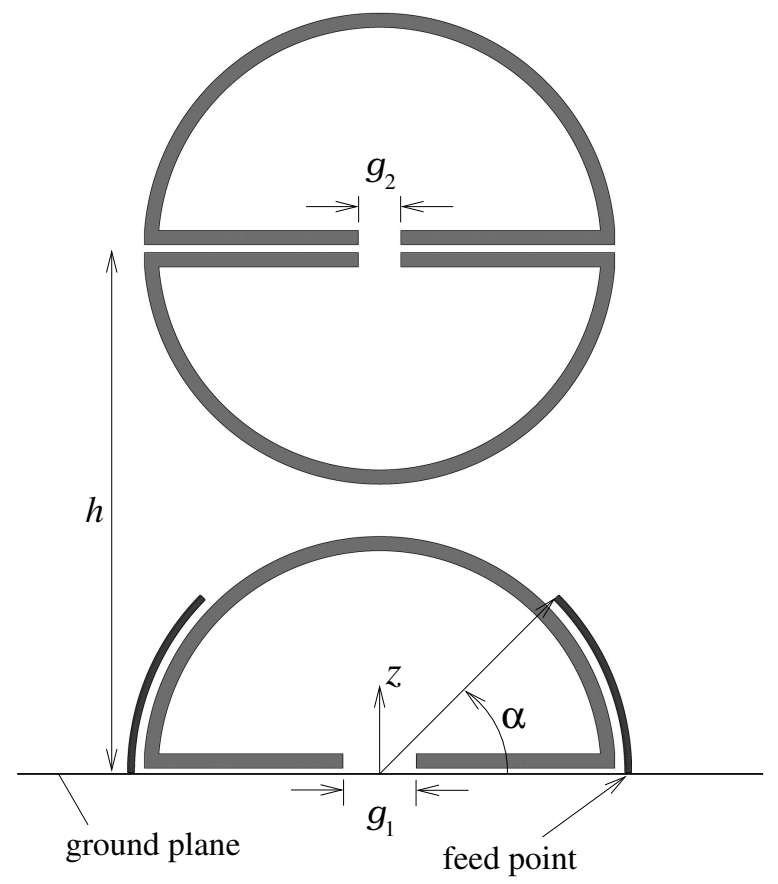

Fig. 4. Two-element superdirective array composed of electrically small resonant magnetic dipole elements on a metal ground plane.

attributed to the fundamental properties of the spherical modes (larger size of the source facilitates excitation of higher-order modes), the high level of the $\mu=0$ modes is caused by asymmetry of the current induced in the CLL by the single active monopole. Indeed, by using a $180^{\circ}$ hybrid to excite the monopoles on both sides of the CLL, it is possible to entirely suppress all even azimuthal modes. However here, we limit our design to a single-port antenna, and therefore the choice of the radius $r_{l}$ is based on a compromise between the bandwidth and the level of the $\mu=0$ modes. For further investigation, we select the $r_{l}$ to be $53 \mathrm{~mm}$, which corresponds to $-37 \mathrm{~dB}$ in the level of the $\mu=0$ modes. In the next section we show that this level can be further reduced in a superdirective array.

\section{Superdirective Array of Magnetic Dipole ELEMENTS}

To create a superdirective array, another electrically small magnetic dipole element is placed in front of the element selected in Section III. Similar to the superdirective arrays composed of resonant electrically small electric monopoles [10], [11], we can expect in our case as well that it is sufficient to feed only one element in the array, while the other one remains passive. To simplify the tuning, we strip the CLL off the curved dipoles and use it as a passive element. Although it is not completely identical to the active element, it is still resonant and therefore yields the dipole radiation pattern. Consequently, the theory of an ideal superdirective array (Section II) composed of elements with identical radiation patterns still applies.

The resulting configuration is sketched in Fig. 4. The passive element is centered at $h=0.2 \lambda_{0}=138 \mathrm{~mm}$ above the ground plane, and its dimensions are identical to those of the CLL of the active element, except the gap $g_{2}$. The latter parameter is 


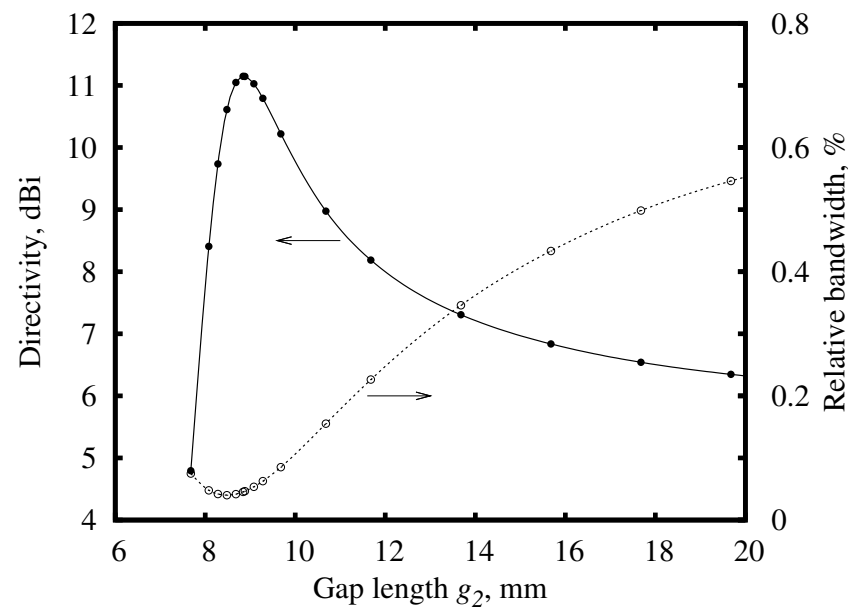

(a)

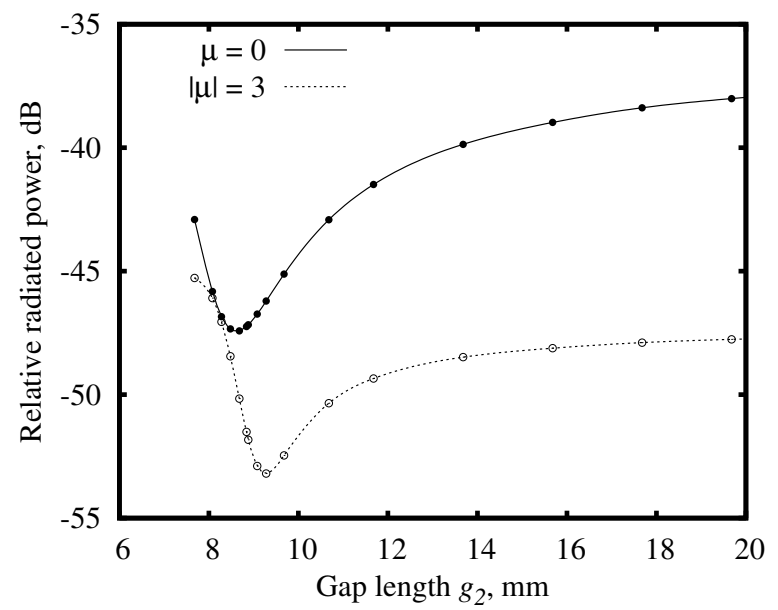

(b)

Fig. 5. Directivity and relative frequency bandwidth (a), relative radiated power of $\mu=0$ and $|\mu|=3$ spherical modes (b) versus the gap width $g_{2}$ of the passive element in the two-element superdirective array (Fig 4).

used to tune the directivity, as illustrated in Fig. 5a, where the variation of the antenna directivity versus the the gap $g_{2}$ is presented. Again, in all cases $g_{1}$ and $\alpha$ are adjusted, so that the resonance input impedance is $50 \mathrm{ohms}$ at $435 \mathrm{MHz}$. We can observe that the maximum directivity occurring at $g_{2}=8.8 \mathrm{~mm}$ is $11.14 \mathrm{dBi}$ (including $3 \mathrm{~dB}$ due to the ground plane), which agrees very well with the value $11.17 \mathrm{~dB}-$ the theoretical prediction found using the procedure described in Section II for an array of two elementary magnetic dipoles spaced by $0.2 \lambda_{0}$.

Typically for a superdirective array, the frequency bandwidth narrows extremely in the vicinity of the maximum directivity, as seen in Fig. 5a. On the other hand, the radiated power of the $\mu=0$ and $|\mu|=3$ spherical modes also minimizes (Fig. 5b) implying that the superdirectivity can effectively suppress the parasitic $(|\mu| \neq 1)$ modes.

Thus, we have shown that a superdirective array with spectral characteristics suitable for a first-order probe can be created using electrically small resonant magnetic dipole elements. The array can be excited using a single actively fed element while the other remains passive.

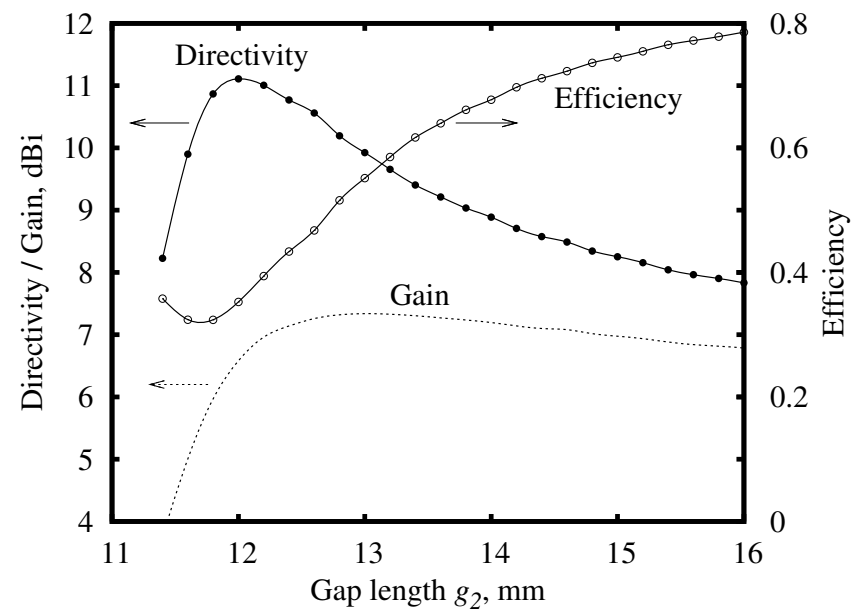

Fig. 6. Directivity, efficiency, and gain versus the gap width $g_{2}$ of the passive element in the two-element superdirective array (Fig 4), when losses are taken into account.

\section{Superdirective First-Order Probe}

Superdirective antennas are usually associated with low efficiency. However, if the directivity is not driven to its extreme value, a reasonable compromise between the directivity and efficiency is possible [11]. This assertion is illustrated in Fig. 6, where the directivity and efficiency are plotted versus the gap width $g_{2}$. It is observed that there is an optimum $g_{2}=13 \mathrm{~mm}$ ensuring the maximum gain of $7.3 \mathrm{~dB}$ corresponding to the directivity of $9.9 \mathrm{~dB}$ and efficiency of $55 \%$.

The simulations are performed with the commercially available software Ansoft HFSS for a realistic design including the dielectric substrate Rogers $\mathrm{RO} 4003 \mathrm{C}$ and losses in all parts of the antenna. The dielectric permittivity $\varepsilon_{\mathrm{r}}$ and the loss tangent $\tan \delta$ of the 60-mil thick substrate are assumed to be 3.55 and 0.0005 , respectively, at $435 \mathrm{MHz}$. The active and parasitic monopoles are now represented by printed strips $1 \mathrm{~mm}$ wide. The width of the CLL strip is $w_{l}=10 \mathrm{~mm}$. Other parameters are fixed as follows: $r_{l}=50 \mathrm{~mm}, g_{1}=20 \mathrm{~mm}, w_{c}=3 \mathrm{~mm}$, $g=2 \mathrm{~mm}, h=130 \mathrm{~mm}$, and $\alpha=14^{\circ}$.

For the prototype to be fabricated, the ground plane has to be finite with the shape preferably circular so that the edge diffraction does not give rise to higher-order azimuthal modes. The size of the ground plane is optimized for maximum front to back ratio resulting in diameter of $720 \mathrm{~mm}$, which corresponds to $1.04 \lambda_{0}$ at $435 \mathrm{MHz}$. The dielectric substrate is fixed vertically by two posts made of Tufnol circular rod $10 \mathrm{~mm}$ in diameter. To keep the resonance frequency at $435 \mathrm{MHz}$ and the input impedance at $50 \mathrm{ohms}$, the effects of the finite ground plane and the supporting posts are compensated by adjusting $g_{1}=22.1 \mathrm{~mm}, g_{2}=14.4 \mathrm{~mm}$, and $\alpha=16^{\circ}$.

The fabricated prototype of the superdirective first-order probe is shown in Fig. 7. One can note that the upper corners of the substrate are cut; this is done rather for aesthetic than performance purposes.

Measurements of the reflection coefficients revealed a shift in the resonance frequency, as shown in Fig. 8. Possible reasons causing the shift have been investigated, and it was found out that the ceramic substrate Rogers RO4003C exhibited 


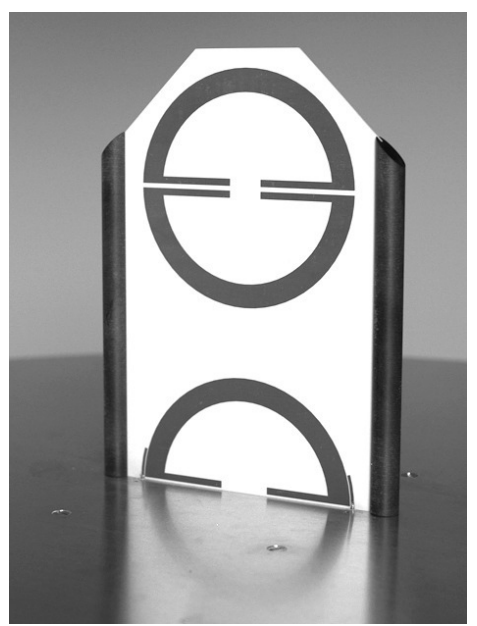

Fig. 7. Fabricated prototype of the two-element superdirective array composed of electrically small resonant magnetic dipole elements.

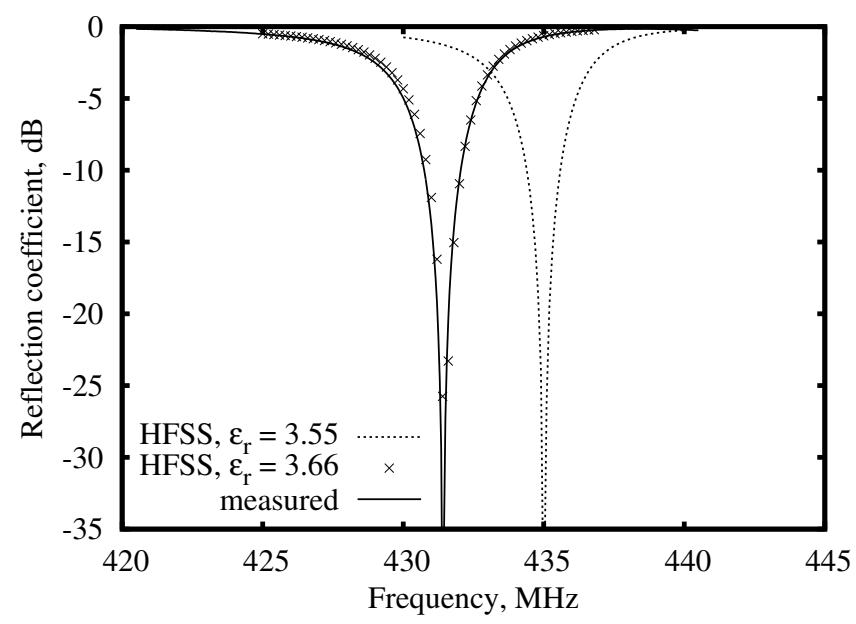

Fig. 8. Predicted and measured reflection coefficient for the fabricated prototype of the two-element superdirective array.

a noticeable anisotropy, that is, the permittivity component tangential to the surface of the substrate $\varepsilon_{\|}$tends to be higher than the normal component $\varepsilon_{\perp}$ [20]. This anisotropy has been reported to affect the resonance frequency of microwave filters [21] as well as printed antennas [22]. In our superdirective array, it is the tangential component $\varepsilon_{\|}$that plays the major role affecting both the capacitive loading of the CLL and the coupling between the monopoles and the CLL. We resimulated the antenna assuming the substrate permittivity to be equal to the tangential permittivity component $\varepsilon_{\|}=3.66$ [22]. The result shown in Fig. 8 is in excellent agreement with the measurements, confirming that it is important to account for the substrate anisotropy when designing resonant printed antennas.

The radiation characteristics of the probe have been measured at the DTU-ESA Spherical Near-Field Antenna Test Facility [5]. The radiation pattern measured at the resonance (431.4 MHz) is presented in Fig. 9. With the directivity of $9.2 \mathrm{~dB}$ and the efficiency of $61 \%$, the gain reaches $7 \mathrm{~dB}$. Within the antenna under test field of view $\left( \pm 30^{\circ}\right)$ the pattern is nearly rotationally symmetric, without oscillations and side-

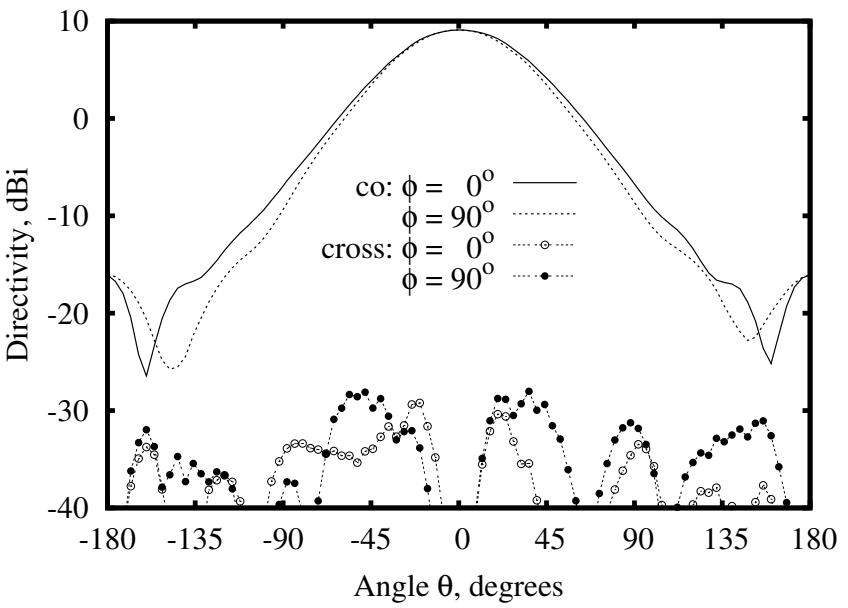

Fig. 9. Measured radiation pattern at 431.4 $\mathrm{MHz}$ for the fabricated prototype of the two-element superdirective array.

lobes. The spherical wave expansion of the radiation pattern shows that the $\mu=0$ and $|\mu|=2$ modes are at $-36 \mathrm{~dB}$ and $-40 \mathrm{~dB}$, respectively, while the higher-order modes $|\mu|>2$ are suppressed even more.

The probe was also measured with a $180^{\circ}$ hybrid exciting the monopoles on both sides of the CLL. As expected, the $\mu=0$ modes were further suppressed to $-39 \mathrm{~dB}$. On the other hand, the hybrid introduced an additional $1 \mathrm{~dB}$ loss into the system.

\section{CONCLUSIONS}

It has been demonstrated, both theoretically and experimentally, how a superdirective array of electrically small resonant magnetic dipole elements can be designed. The array utilizes a single active element located directly on a metal ground plane, while identical passive elements are placed in front of it in a Yagi-Uda-antenna manner. Such an array can potentially exhibit directivity of $11.5 \mathrm{dBi}, 15.2 \mathrm{dBi}$, and $17.8 \mathrm{dBi}$ (including $3 \mathrm{~dB}$ due to the ground plane), if composed of two, three, and four closely spaced elementary magnetic dipoles, respectively. ${ }^{3}$

In our study, we have used a two-gap capacitively loaded loop as a resonant element. It is driven directly through the ground plane by a $50-\mathrm{ohm}$ coaxial cable, and its symmetry with respect to the two orthogonal vertical planes ensures the domination of the $|\mu|=1$ modes in the spherical wave spectrum. The presented numerical results show that an identical passive element placed in front of the active one not only adds the superdirective properties to the antenna but also improves the purity of the $|\mu|=1$ modes. The maximum directivity found by numerical simulations for such two-element array agrees very well with the theoretical prediction.

A $435 \mathrm{MHz}$ prototype of the two-element superdirective array has been fabricated and measured. Measurement results

\footnotetext{
${ }^{3}$ As discussed in Section II-B, the 2-, 3-, or 4-element array of magnetic dipoles over a PEC ground plane is equivalent to a symmetric array of 3,5 , or 7 magnetic dipoles in free space, respectively. Due to the symmetry, the latter has the maximum directivity in two end-fire directions, and thus should not be confused with an array of dipoles in free space optimized for maximum directivity in one end-fire direction, as for example those in [11].
} 
show that the antenna is well matched to 50 ohms, the directivity is $9.2 \mathrm{dBi}$, whereas the parasitic $(|\mu| \neq 1)$ azimuthal modes are suppressed to at least $-36 \mathrm{~dB}$. This implies that the obtained radiation characteristics are comparable to those of conical horns - the first-order probes typically utilized at higher frequencies.

The obtained frequency bandwidth is narrow. On the other hand, it is often sufficient to measure only at the central frequency in a band of interest. When measurements at several frequencies are required, dedicated superdirective probes for each frequency can easily be fabricated, since the proposed probe is very cheap and technologically simple.

In particular, we used the fabricated superdirective array as a first-order probe to obtain a reference measurement of a representative antenna under test as part of the validation campaign for the DTU-ESA wideband dual-polarized higherorder 0.4-1.2 GHz probe [7], [23]. The latter was recently developed at the Technical University of Denmark (DTU) within an ESA project in preparation to forthcoming measurements of different antennas for future ESA missions at frequencies as low as $400 \mathrm{MHz}$, such as BIOMASS [24].

\section{REFERENCES}

[1] J. E. Hansen, Spherical Near-Field Antenna Measurements. London, U.K.: Peter Peregrinus, 1988.

[2] T. Laitinen, S. Pivnenko, J. M. Nielsen, and O. Breinbjerg, "Theory and practice of the FFT/matrix inversion technique for probe-corrected spherical near-field antenna measurements with high-order probes," IEEE Trans. Antennas Propagat., vol. 58, no. 8, pp. 2623-2631, 2010.

[3] T. B. Hansen, "Complex-point dipole formulation of probe-corrected cylindrical and spherical near-field scanning of electromagnetic fields," IEEE Trans. Antennas Propagat., vol. 57, no. 3, pp. 728-741, March 2009.

[4] —_ "Spherical near-field scanning with higher-order probes," IEEE Trans. Antennas Propagat., vol. 59, no. 11, pp. 4049-4059, November 2011.

[5] DTU-ESA Spherical Near-Field Antenna Test Facility. Homepage, http: //www.dtu.dk/centre/ems/English/research/dtu_esa_facility.aspx.

[6] O. S. Kim and O. Breinbjerg, "Metamaterial-inspired first-order probe for spherical near-field antenna measurements," in 3rd International Topical Meeting on Nanophotonics and Metamaterials, NANOMETA2011, Conference Abstracts, vol. 35A, Seefeld, Tirol, 3-6 January 2011.

[7] O. S. Kim, S. Pivnenko, and O. Breinbjerg, "Wideband scalable probe for spherical near-field antenna measurements," in Proc. 5th European Conference on Antennas and Propagation (EuCAP'2011), Rome, Italy, 2011, pp. $1781-1785$.

[8] A. I. Uzkov, "An approach to the problem of optimum directive antennae design," Comptes Rendus de l'Académie des Sciences de l'URSS, vol. 53, no. 1 , pp. 35-38, 1946.

[9] E. E. Altshuler, T. H. O'Donnell, A. D. Yaghjian, and S. R. Best, "A monopole superdirective array," IEEE Trans. Antennas Propagat., vol. 53, no. 8, pp. 2653 - 2661, August 2005.

[10] T. H. O'Donnell and A. D. Yaghjian, "Electrically small superdirective arrays using parasitic elements," in Proc. IEEE Antennas Propag. Soc. Int. Symp., Albuquerque, NM, USA, July 2006, pp. 3111 -3114.

[11] A. D. Yaghjian, T. H. O'Donnell, E. E. Altshuler, and S. Best, "Electrically small supergain end-fire arrays," Radio Science, vol. 43, no. 3, p. RS3002, 2008.

[12] S. Lim and H. Ling, "Design of electrically small Yagi antenna," Electron. Lett., vol. 43, no. 5, pp. 3-4, 12007.

[13] A. D. Yaghjian and H. R. Stuart, "Increasing the bandwidth of electrically small supergain antennas using low-Q electric dipoles," in Proc. 5th European Conference on Antennas and Propagation (EuCAP'2011), Rome, Italy, April 2011, pp. 2720-2723.

[14] H. Bach and J. E. Hansen, "Uniformly spaced arrays," in Antenna Theory Part 1, R. E. Collin and F. J. Zucker, Eds. New York: McGraw-Hill, 1969.
[15] S. Hrabar, Z. Eres, and J. Bartolic, "Capacitively loaded loop as basic element of negative permeability meta-material," in Proc. 32nd Eur. Microwave Conf., Milan, Italy, September 24-26 2002.

[16] P. Jin and R. Ziolkowski, "Metamaterial-inspired, electrically small Huygens sources," IEEE Antennas Wireless Propagat. Lett., vol. 9, pp. 501-505, 2010.

[17] O. S. Kim and O. Breinbjerg, "Miniaturised self-resonant split-ring resonator antenna," Electron. Lett., vol. 45, no. 4, pp. 196-197, Feb. 2009.

[18] O. S. Kim, "Low-Q electrically small spherical magnetic dipole antennas," IEEE Trans. Antennas Propagat., vol. 58, no. 7, pp. 2210-2217, July 2010

[19] E. Jørgensen, O. S. Kim, P. Meincke, and O. Breinbjerg, "Higher order hierarchical legendre basis functions in integral equation formulations applied to complex electromagnetic problems," in Proc. IEEE Antennas and Propagation Soc. Int. Symp., vol. 3A, Washington DC, Jul. 2005, pp. 64-67.

[20] V. N. Levcheva, B. N. Hadjistamov, and P. I. Dankov, "Two-resonator method for characterization of dielectric substrate anisotropy," Bulg. J. Phys., vol. 35, no. 1, pp. 33-52, 2008.

[21] J. C. Rautio, B. J. Rautio, S. Arvas, A. F. Horn III, and J. W. Reynolds, "The effect of dielectric anisotropy and metal surface roughness," in Proc. Asia-Pacific Microwave Conference Proceedings (APMC), 2010, pp. $1777-1780$.

[22] P. I. Dankov, M. I. Kondeva, and S. R. Baev, "Influence of the substrate anisotropy in the planar antenna simulations," in Proc. of International Workshop on Antenna Technology (iWAT2010), Lisbon, Portugal, 2010.

[23] O. S. Kim, S. Pivnenko, and O. Breinbjerg, "0.4-1.2 GHz hybrid AlCFRP open-boundary quad-ridge horn," in Proc. 33rd ESA Antenna Workshop, ESTEC, Noordwijk, the Netherlands, 18-21 October 2011.

[24] T. Le Toan, S. Quegan, M. Davidson, H. Balzter, P. Paillou, K. Papathanassiou, S. Plummer, F. Rocca, S. Saatchi, H. Shugart, and L. Ulander, "The BIOMASS mission: Mapping global forest biomass to better understand the terrestrial carbon cycle," Remote Sensing of Environment, vol. 115 , no. 11 , pp. 2850-2860, 2011.

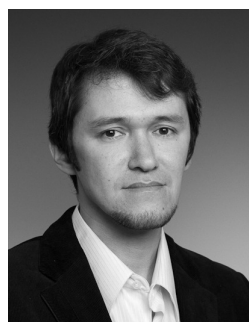

Oleksiy S. Kim received the M.S. and Ph.D. degrees from the National Technical University of Ukraine, Kiev, in 1996 and 2000, respectively, both in electrical engineering. In 2000, he joined the Antenna and Electromagnetics Group at the Technical University of Denmark (DTU). He is currently an associate professor with the Department of Electrical Engineering, Electromagnetic Systems, DTU.

His research interests include electrically small antennas, computational electromagnetics, metamaterials, photonic bandgap and plasmonic structures.

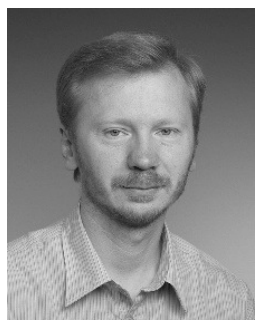

Sergey Pivnenko (M'98) was born in Kharkiv, Ukraine, in 1973. He received the M.Sc. and Ph.D. degrees in electrical engineering from Kharkiv National University, Ukraine, in 1995 and 1999, respectively. From 1998 to 2000, he was a Research Fellow at the Radiophysics Department, Kharkiv National University. In 2000, he joined the Electromagnetic Systems Group, Department of Electrical Engineering, Technical University of Denmark, where he now works as an Associate Professor and operates the DTU-ESA Spherical Near-Field Antenna Test Facility. Since 2000, he participated to several research projects related to design, development and characterization of satellite antennas, development of new near-field probes and probe correction techniques for near-field antenna measurements. From 2004-2007, he was a work package leader in the European Union network "Antenna Center of Excellence" where he was responsible for antenna measurement facility comparisons and participated to the other activities related to antenna measurements. He is the author or coauthor of more than 100 journal and conference papers. His research interests include antenna measurement techniques, antenna analysis and design. 


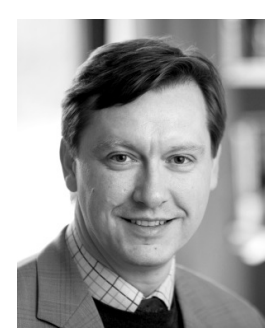

Olav Breinbjerg was born in Silkeborg, Denmark on July 16, 1961. He received the M.Sc. and Ph.D. degrees in electrical engineering from the Technical University of Denmark (DTU) in 1987 and 1992, respectively. Since 1991 he has been on the faculty of the Department of Electrical Engineering as Assistant Professor (1991-1995), Associate Professor (1995-2005), and Full Professor (since 2006). He is now Head of the Electromagnetic Systems Group and the DTU-ESA Spherical Near-Field Antenna Test Facility. Olav Breinbjerg was a Visiting Scientist at Rome Laboratory, Massachusetts, USA in the fall of 1988, a Fulbright Research Scholar at the University of Texas at Austin, Texas, USA in the spring of 1995, and a Visiting Professor at the University of Siena, Italy in the spring of 2011. He has been, or is, the main supervisor of $12 \mathrm{Ph} . \mathrm{D}$. projects. Olav Breinbjerg's research is generally in applied electromagnetics and particularly in antennas, antenna measurements, computational techniques and scattering - for applications in wireless communication and sensing technologies. He is the author or co-author of more than 45 journal papers, 150 conference papers, and 75 technical reports. Olav Breinbjerg received a US Fulbright Research Award in 1995. Also, he received the 2001 AEG Elektron Foundation's Award in recognition of his research in applied electromagnetics. Furthermore, he received the 2003 DTU Student Union's Teacher of the Year Award for his course on electromagnetics. 